\title{
In vivo assessment of cerebrospinal fluid efflux to nasal mucosa in humans
}

\begin{abstract}
Erik Melin $^{1}$, Per Kristian Eide ${ }^{2,3 \bowtie}$ \& Geir Ringstad ${ }^{4}$
Extra-vascular molecular clearance routes from the brain and cerebrospinal fluid (CSF) remain insufficiently characterized in humans. Animal studies consistently suggest that the cribriform plate and nasal lymphatic vessels are crucial for molecular clearance from CSF. In this study, we aimed to examine human in vivo transport of a CSF tracer from CSF to nasal mucosa. We hypothesised a CSF tracer would enrich in nasal mucosa provided that nasal lymphatic drainage has a significant role in CSF molecular clearance. Consecutive magnetic resonance imaging during $48 \mathrm{~h}$ after intrathecal administration of a tracer (gadobutrol) was performed in 24 patients. Despite a strong enrichment of CSF tracer in CSF spaces nearby the cribriform plate, there was no significant enrichment of CSF tracer in nasal mucosa, as measured in superior, medial and inferior turbinates, or in the nasal septum. Therefore, this in vivo study questions the importance of CSF drainage to the human nasal mucosa and emphasizes the need of further human studies.
\end{abstract}

There is limited knowledge about molecular efflux routes from cerebrospinal fluid (CSF) to lymphatic pathways in humans. Based on studies of a range of animal species, drainage to nasal mucosa via the cribriform plate is consistently shown to represent a major drainage route ${ }^{1-10}$. Hence, this route may be hypothesised to be of equal importance in humans. However, only one in vivo human study, utilizing radionuclide tracers, has been able to demonstrate possible clearance of molecules to the nasal turbinates, presumably via the cribriform plate ${ }^{11}$.

The recent reports of lymphatic vessels lining the dural sinuses ${ }^{12,13}$, and the possible connection between meningeal lymphatics and the glymphatic system ${ }^{14}$, have sparked a renewed interest in lymphatic drainage routes from CSF spaces. Experimentally induced impairment of meningeal lymphatic vessels suggests that lymphatic drainage is crucial for brain molecular clearance ${ }^{13,15}$, indicating a role of lymphatic drainage failure in neurodegeneration caused by the accumulation of neurotoxic molecules in brain tissue. Recently, our group used magnetic resonance imaging (MRI) to demonstrate the accumulation of a MRI contrast agent administered intrathecally in the human parasagittal dura, a tissue possibly serving as an intermediate step towards meningeal lymphatics, paralleled by efflux through neuroforamina at the skull base ${ }^{16}$. The methodology has yet not been used to assess whether this CSF tracer is cleared to nasal lymphatic efflux pathways.

In this study, we have therefore examined the efflux of intrathecal gadobutrol to nasal mucosa utilizing multi-phase, long-term MRI. We hypothesised that the MRI contrast agent gadobutrol, when administered intrathecally, enriches in nasal mucosa.

\section{Results}

Patients. The study included 24 patients examined with consecutive MRI scans before and after intrathecal administration of gadobutrol for work-up of tentative CSF circulation disorders. Patient data are given in Table 1.

CSF tracer enrichment in CSF spaces. The tracer was detected in CSF close to the cribriform plate in all patients. There was a highly significant $(\mathrm{P}<0.001)$ enrichment of CSF tracer in the subarachnoid space $(\mathrm{SAS})$ adjacent to the straight gyrus with peak enrichment after 3-6 h (Fig. 1; Supplementary Table S1).

${ }^{1}$ Department of Radiology, Østfold Hospital Trust, Grålum, Norway. ${ }^{2}$ Department of Neurosurgery, Oslo University Hospital-Rikshospitalet, Oslo, Norway. ${ }^{3}$ Institute of Clinical Medicine, Faculty of Medicine, University of Oslo, Oslo, Norway. ${ }^{4}$ Department of Radiology and Nuclear Medicine, Oslo University Hospital-Rikshospitalet, Oslo, Norway. ${ }^{\circledR}$ email: p.k.eide@medisin.vio.no 


\begin{tabular}{|l|l|}
\hline \multicolumn{1}{|l|}{} & Total material \\
\hline $\mathrm{N}$ & 24 \\
\hline Age (years) & $41 \pm 15$ \\
\hline Gender $($ female/male) & $18 / 6$ \\
\hline BMI $\left(\mathrm{kg} / \mathrm{m}^{2}\right)$ & $28 \pm 5$ \\
\hline Clinical indication for MRI & \multicolumn{2}{|l}{} \\
\hline iNPH & $1(4 \%)$ \\
\hline Tentative spontaneous intracranial hypotension & $3(13 \%)$ \\
\hline Arachnoid cyst & $7(29 \%)$ \\
\hline Pineal cyst & $6(25 \%)$ \\
\hline Idiopathic intracranial hypertension & $7(29 \%)$ \\
\hline
\end{tabular}

Table 1. Patient material. Data are given as numbers (percentage in parenthesis) and mean \pm standard deviation.

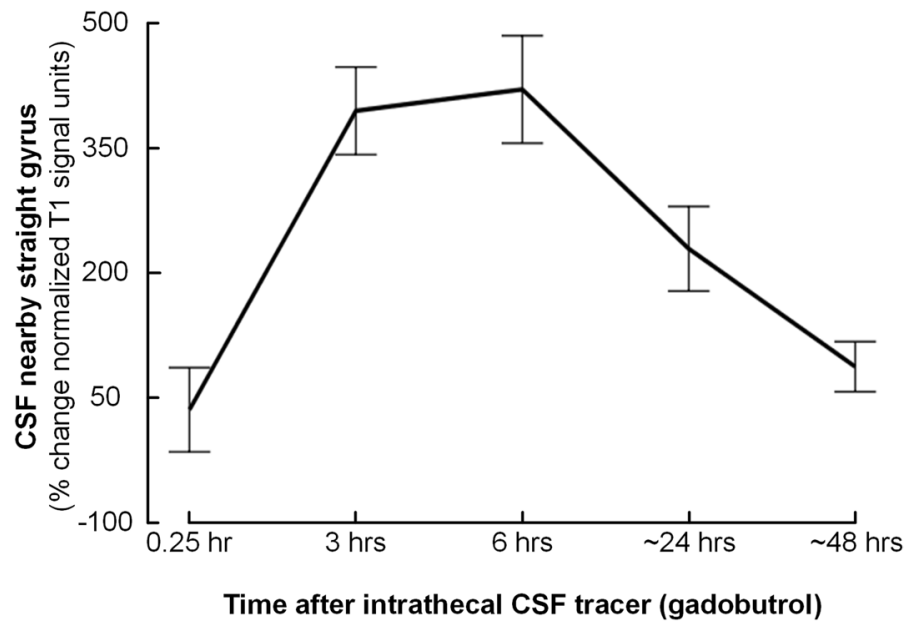

Figure 1. CSF tracer enrichment in CSF spaces. The percentage change in signal unit ratios over time is shown for CSF nearby the straight gyrus. The plot presents average percentage changes from the pre-tracer images with $95 \%$ confidence interval for every time point. Significance levels for the different regions of interest measured are presented in Table 2. There was a strongly significant enrichment of CSF tracer in this CSF space.

CSF tracer enrichment inferior to the cribriform plate. Tracer was detected as minute tubular structures inferior to the cribriform plate 3-6 h after injection in 11 of 24 patients. Figure 2 is a post-processed image illustrating the distribution of tracer in one of the patients.

CSF tracer enrichment in nasal mucosa. The CSF tracer enrichment in different regions of interest within nasal mucosa at group level is presented in Fig. 3. The percentage change in signal unit ratio was not significant at any time point in any of the nasal cavity structures: (Fig. 3a) superior turbinate, (Fig. 3b) middle turbinate, (Fig. 3c) inferior turbinate and (Fig. 3d) nasal septum (see Supplementary Table S2-S5). Even though the signal unit ratio tended to increase $3 \mathrm{~h}$ after tracer administration for the middle and the inferior turbinates and after $6 \mathrm{~h}$ for the superior turbinate, the variation was large and not statistically significant.

CSF tracer enrichment in brain parenchyma. There was a highly significant $(\mathrm{P}<0.001)$ enrichment of CSF tracer within the grey matter of the straight gyrus (Fig. 4a; Supplementary Table S6), as well as in the deep white matter of the frontal lobe (Fig. 4b; Supplementary Table S7).

Safety of intrathecal contrast-enhanced MRI. No anaphylactic reactions, other serious adverse reactions or complications were noticed during or after the exams.

\section{Discussion}

The main result of this study was that no CSF tracer could be detected in human nasal mucosa in vivo, raising the question of the relative importance of CSF clearance to lymphatic vessels in the nasal mucosa.

Lumbar intrathecal administration of the MRI contrast agent gadobutrol, serving as CSF tracer, has previously been shown to facilitate robust enrichment in the intracranial SAS compartment ${ }^{17}$. The time from injection until CSF tracer was present in cisterna magna was $20 \pm 23 \mathrm{~min}$ in humans ${ }^{18}$. Enrichment of tracer in CSF is a 


\begin{tabular}{|l|c|}
\hline Region of interest & Significance \\
\hline Superior turbinates & 0.252 \\
\hline Middle turbinates & 0.302 \\
\hline Inferior turbinates & 0.135 \\
\hline Nasal septum & 0.676 \\
\hline Straight gyrus & $<0.001$ \\
\hline White matter in frontal lobes & $<0.001$ \\
\hline SAS adjacent to straight gyri & $<0.001$ \\
\hline Left side & 0.045 \\
\hline Superior turbinate & 0.322 \\
\hline Middle turbinate & 0.172 \\
\hline Inferior turbinate & 0.68 \\
\hline Nasal septum & $<0.001$ \\
\hline Straight gyrus & 0.001 \\
\hline White matter in frontal lobe & $<0.001$ \\
\hline SAS adjacent to straight gyrus & 0.725 \\
\hline Right side & 0.39 \\
\hline Superior turbinate & 0.1 \\
\hline Middle turbinate & $<0.001$ \\
\hline Inferior turbinate & $<0.001$ \\
\hline Nasal septum & \multicolumn{2}{|l|}{} \\
\hline Straight gyrus & \\
\hline White matter in frontal lobe & \\
\hline SAS adjacent to straight gyrus & \multicolumn{2}{|l|}{} \\
\hline
\end{tabular}

Table 2. Significance for percentage change in signal unit ratios for the different regions of interest. SAS subarachnoid space. Significance level was determined by mixed model analysis.

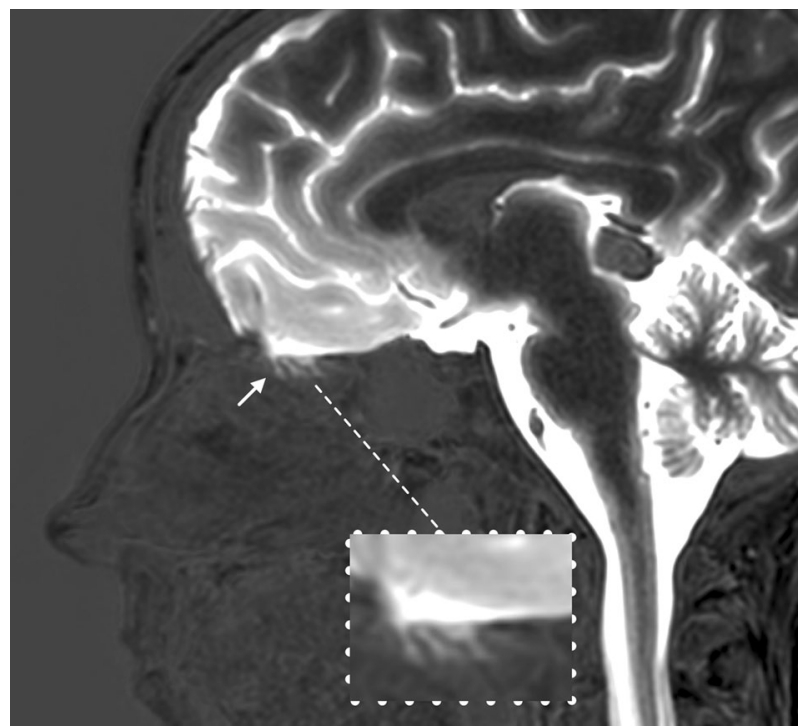

Figure 2. Subtraction magnetic resonance image visualizing tracer immediately below the cribriform plate. The illustration constitutes of a combination of T1-GRE images obtained at different time points: a summation of pre-contrast and 48 h' post-contrast time points (with no or small amounts of CSF tracer) were subtracted from the 3- and 6-h' time points (with high concentrations of tracer in CSF) to visualize the distribution of tracer. In this example, narrow streaks of tracer are depicted immediately below the cribriform plate (arrow). No tracer enhancement in the nasal mucosa can be readily seen and was confirmed by the ROI-analysis through all time points at group level. 
(a)

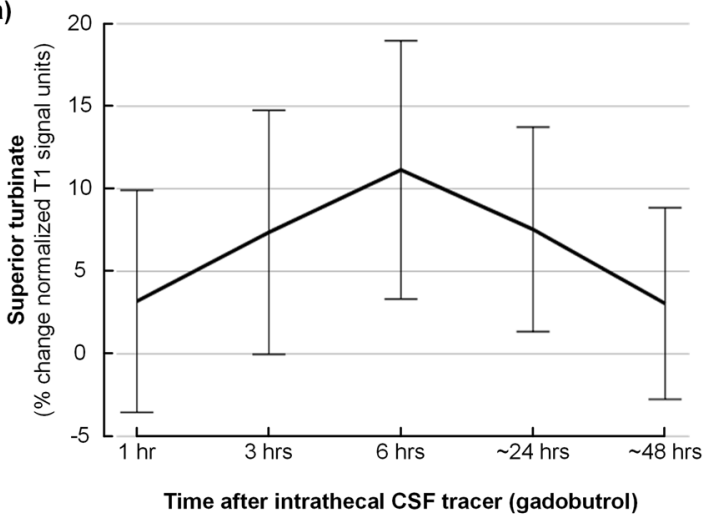

(b)

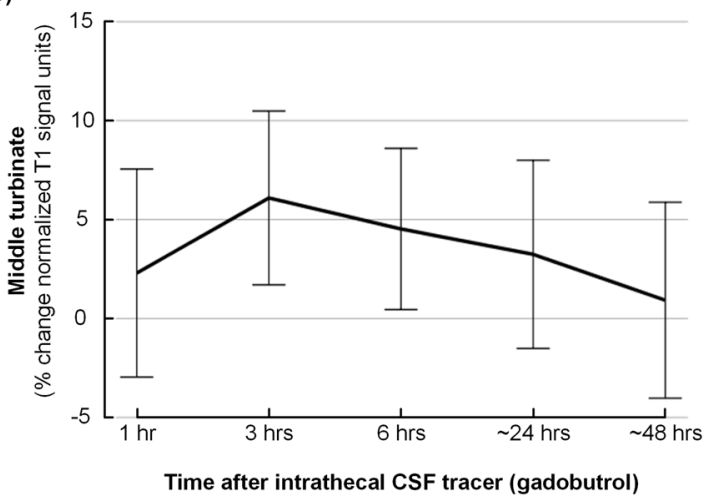

(c)

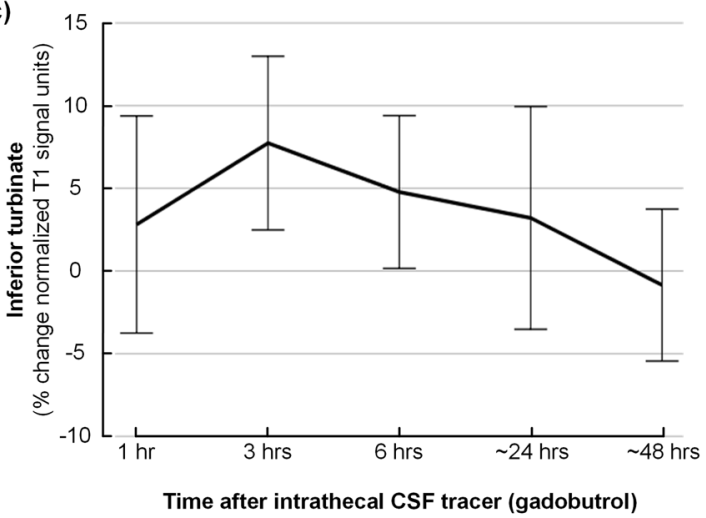

(d)

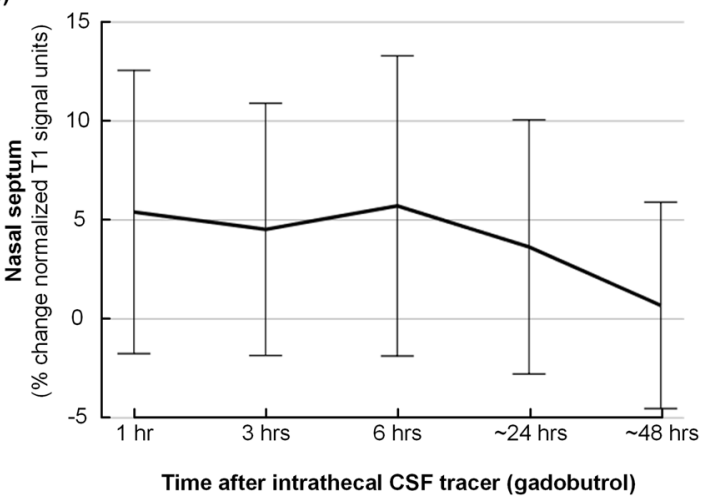

Figure 3. CSF tracer enrichment in nasal mucosa. The percentage change in signal unit ratios over time is shown for (a) superior turbinate, (b) middle turbinate, (c) inferior turbinate, and (d) nasal septum. The plots present average percentage changes from the pre-tracer images with $95 \%$ confidence interval for every time point. Significance levels for the different regions of interest measured are shown in Table 2. No significant enrichment of CSF tracer was observed in these regions.

(a)

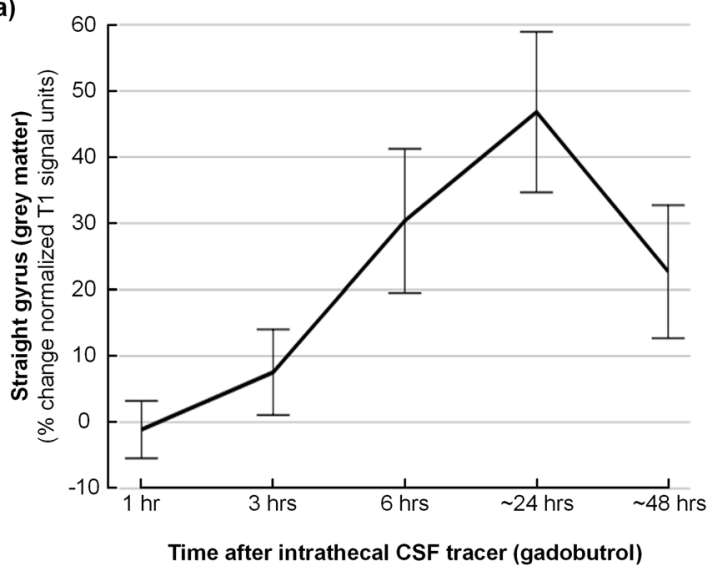

(b)

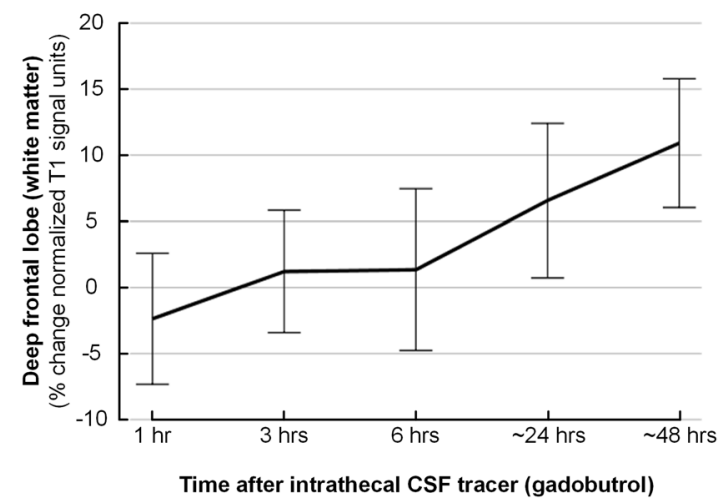

Figure 4. CSF tracer enrichment in brain parenchyma. The percentage change in signal unit ratios over time is shown for (a) straight gyrus and (b) deep white matter of the frontal lobe. The plots present average percentage changes from the pre-tracer images with $95 \%$ confidence interval for every time point. P-values for the different regions of interest measured are presented in Table 2, revealing significant CSF tracer enrichment within the brain parenchyma.

requirement for enhancement in nearby cerebral structures ${ }^{17,19,20}$ as well as in parasagittal dura ${ }^{16}$. The present observations confirmed entry of tracer into the adjacent straight gyrus and strong enrichment of tracer in the SAS close to the cribriform plate. Tracer was also detected as minute tubular structures inferior to the cribriform plate in 11/24 patients (Fig. 2). Enrichment within nasal mucosa could be expected if efflux via the cribriform plate had a major role in human subjects. 
Impaired clearance of toxic metabolites from CNS is considered an important mechanism behind neurodegenerative disease $\mathrm{e}^{21-25}$. Recent discoveries have highlighted the perivascular route for clearance of macromolecules from the brain, such as $\beta$-amyloid and tau ${ }^{14,24,26}$, and meningeal lymphatic vessels seem to play an important role in brain and CSF clearance ${ }^{12,13,15,16,27,28}$. The dural lymphatic drainage route may be particularly important for a particularly toxic amyloid subtype ${ }^{29}$. The arachnoid is commonly considered as a barrier between CSF and the dura mater ${ }^{30}$. How molecules from CSF reach meningeal lymph vessels is not fully described, but in a previous study we detected a CSF tracer in parasagittal dura challenging the concept of an impermeable arachnoid ${ }^{16}$.

The tracer used in this study, gadobutrol, is of low molecular weight (604 Da), distributes freely in the CSF due to its hydrophilic properties and does not cross the blood-brain-barrier. As so, this MRI-tracer should be expected to follow extra-vascular clearance routes for molecules lacking specific transporters ${ }^{31}$. The movement of the tracer intracranially was assessed by our group in other studies ${ }^{16,17,19,32}$ and the tracer was also detected in the cervical lymph nodes after intrathecal administration ${ }^{33}$. However, molecules of low weight $(<5,000 \mathrm{Da})$ may also be absorbed by capillaries in the nasal mucosa ${ }^{5}$ possibly affecting the detectable amount of tracer in the area. Detection of the tracer in both deep cerebral white matter and in the straight gyrus of the frontal lobes confirmed that the applied T1-BB sequence enables for detection of tracer in limited amounts with good sensitivity.

In this study, we detected the CSF tracer as minute tubular structures inferior to the cribriform plate in 11/24 patients. This could represent tracer along perineural spaces, in lymphatic vessels or in the interstitial space ${ }^{4}$, or even tracer in arachnoid villi ${ }^{34}$. Perineural spread of tracer through the cribriform plate to nasal mucosa has previously been described in human ${ }^{10,34}$. In Johnston et al. ${ }^{10}$ a tracer injected in the SAS did indeed reach the lymphatic vessels of the nasal mucosa, both in animals and in one human, but no connection to lymphatic vessels was detected by Lowhagen et al. ${ }^{34}$. These studies provide good anatomical descriptions of the area, but do not necessarily reflect physiological conditions as they are performed post-mortem.

Our study findings contradicts De Leon et al., who utilized dynamic positron emission tomography (PET) to measure CSF clearance in humans and found significant levels of CSF tracer in the superior turbinates ${ }^{11}$. The PET technique used is probably more sensitive compared to MRI, but the image resolution of $4,5 \mathrm{~mm}$ is inferior to MRI ( $1 \mathrm{~mm})$. The superior turbinates in humans are small structures, and the ROI used for superior turbinates in the PET-study may potentially have included adjacent areas. The ROI was also placed $5.2 \mathrm{~mm}$ inferior to the frontal lobe to avoid partial-volume effects from the brain, nevertheless, partial volume effects from CSF may have affected the measurements. Our results do confirm tracer inferior to the cribriform plate in a substantial proportion of patients (11/24) (Fig. 2), however, drainage to the nasal mucosa itself was not detected. The evidence of drainage to the middle turbinates provided with PET may be due to a higher sensitivity for detection of tracer in limited amounts. As molecules of low weight may to some extent be absorbed by capillaries before entering lymphatic vessels in nasal mucosa ${ }^{5}$, we anticipate such "leakage" of tracer not to be very different in these contradictive studies, as De Leon et al. also used molecules with low weight $(<328 \mathrm{Da})$.

Both animal and human studies suggest transport of molecules in the opposite direction, that is, from nasal mucosa to CSF, following anatomical pathways along the olfactory and trigeminal nerves bypassing the blood-brain barrier ${ }^{35}$. Substances have been detected in CSF within $30 \mathrm{~min}$ after intranasal administration ${ }^{36}$. One plausible theoretical explanation for such rapid transport is extracellular (perineural or paravascular) convective flow ${ }^{37}$.

From an anatomical point of view, the human bipedal position, and the small olfactory area, which may be considered to be of a rather rudimentary size compared to rodents ${ }^{38,39}$, could have an impact on speciesdependent differences in trans-nasal CSF molecular efflux. We, therefore, hypothesise that CSF molecular efflux through other foramina in the skull base and meningeal lymphatics ${ }^{16,27,30}$ could be of more importance in humans compared to many other species. Other postulated molecular efflux routes are via the arachnoid villi to dural venous sinuses ${ }^{40}$ and across the blood-brain barrier ${ }^{41}$.

This study included patients with various CSF-disorders (Table 1). 13/24 patients were examined for intracranial cysts not adjacent to the cribriform plate. It is unlikely that these patients' efflux systems in any way would be compromised or affected by the cysts. $8 / 24$ were examined for idiopathic normal pressure hydrocephalus (iNPH) or idiopathic intracranial hypertension. As the pathophysiology of these latter disorders is not understood, the CSF dynamics in these patients could possibly be affected. 3/24 were examined for tentative spontaneous intracranial hypotension. No CSF leakage was seen in these patients, but as for iNPH and idiopathic intracranial hypertension, these patients present with pathology that potentially could have affected the results.

The temporal resolution of imaging was low, particularly for late scans. However, MRI scanning was continued throughout a considerable time frame and beyond the time of peak tracer enhancement in CSF. It seems therefore unlikely that mucosal enrichment followed by an expectedly slow molecular escape should have occurred entirely in intervals between MRI scans.

The connections between the SAS, perineural spaces, lymphatic vessels and interstitial spaces in the nasal mucosa are not clarified ${ }^{4}$. Visualization of individual lymphatic vessels or perineural spaces in the nasal mucosa using MRI cannot be expected due to the MR image resolution $(1 \times 1 \times 1 \mathrm{~mm})$; the circular ROIs were positioned to measure from representative samples of the mucosa/submucosa covering perineural spaces, lymphatic vessels and the interstitial space. The concentration of the tracer in nasal mucosa would be expected to be lower than in the CSF after dilution from lymph and interstitial fluid in the nasal mucosa. Artefacts from both movement and surrounding areas may have decreased the signal-to-noise ratio and confounded the measurements even if obvious artefacts were avoided and images disturbed by motion were excluded. To this end, our negative result could be explained by low concentrations of tracer undetectable by the methodology utilized in this study.

For some measurements, the signal intensity in the CSF, nasal mucosa and brain parenchyma seemingly declined after contrast agent administration compared to pre-contrast images (Figs. 3, 4 and 5). Since this is implausible, the most likely explanation for this is artefacts affecting the signal within any of the ROIs, including the reference ROIs and the ROIs in the CSF, nasal mucosa and brain parenchyma. 


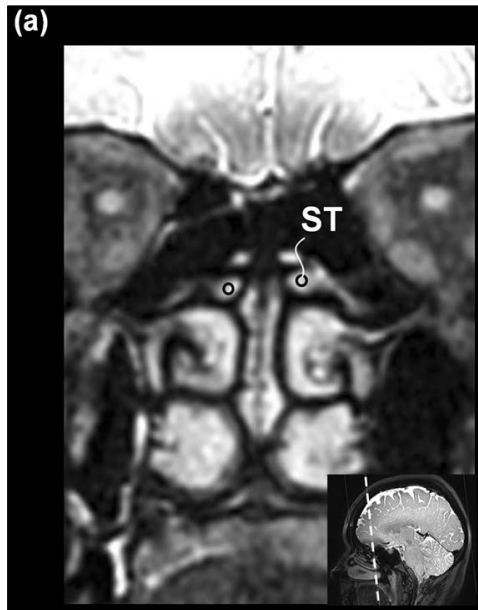

(c)

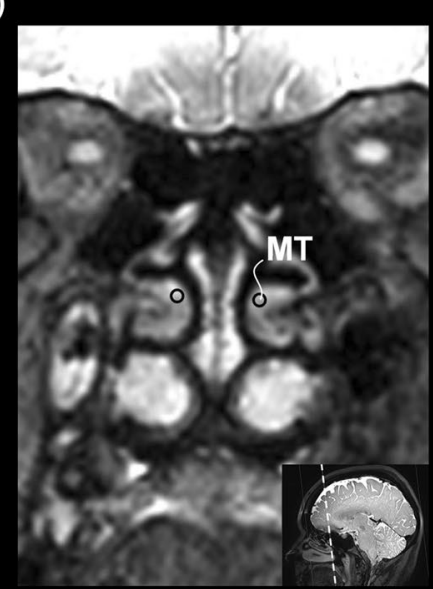

(e)

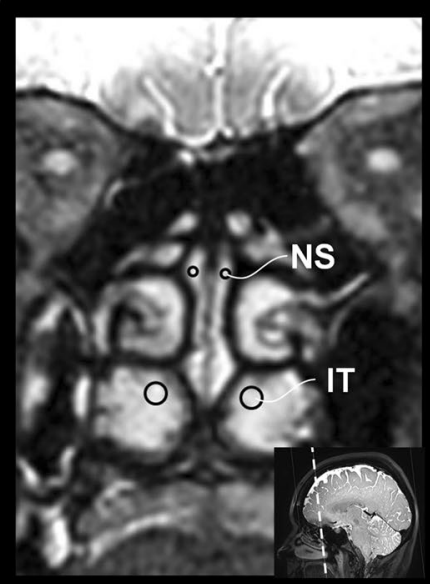

(b)

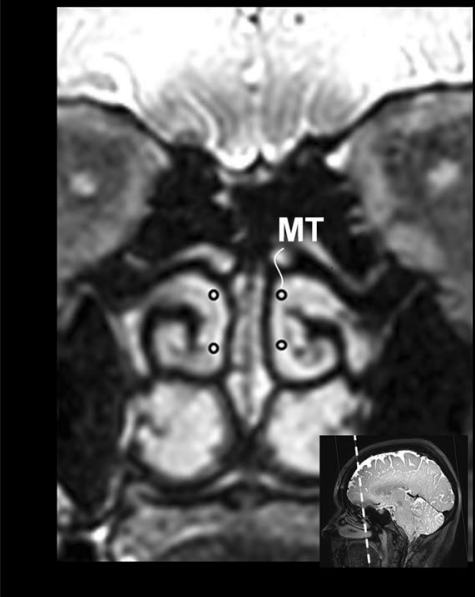

(d)

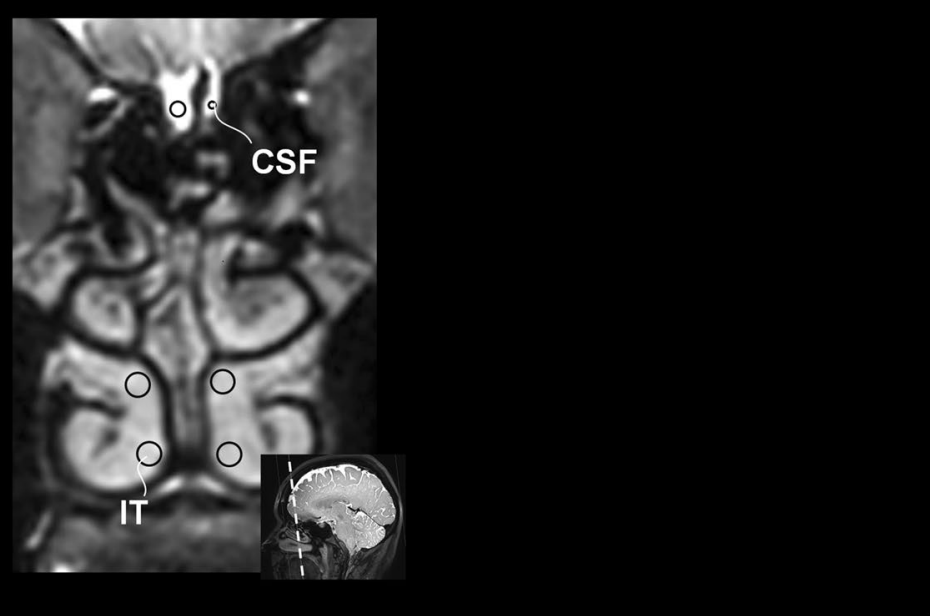

Figure 5. Slice sections and measurement areas on T1-weighted blood suppressed magnetic resonance images. Coronal images of nasal mucosa illustrate the various regions of interest (ROIs) assessed in this study; each ROI is indicated by a circle. The slice section is shown in the lower right corner of each image. Regions of interest indicated by a circle are shown for the following structures (a) superior turbinate (ST), (b) middle turbinate (MT), (c) middle turbinate (MT) here illustrated by the posterior part, (d) inferior turbinate (IT), also showing CSF nearby straight gyri, (e) nasal septum (NS) and posterior part of inferior turbinate (IT).

Diurnal volume fluctuations of the nasal mucosa derive from dilation and constriction of the venous cavernous tissue in the turbinates ${ }^{42}$. Therefore, ROI locations had to be manually adapted to local volume changes to avoid misregistration and partial volume effects from adjacent regions, particularly from the airways. The volume differences represent to some extent differences in blood volume. The intrinsic signal in nasal mucosa could thus potentially change for other reasons than the enrichment of tracer. The volume differences in the nasal mucosa also limited the possible regions to include in the measurements. On the other hand, volume changes would be random, and should not produce a systematic effect of the changes in MRI signal units in one direction. Even though manual placement of ROIs may not be optimal, and potentially biased, this was considered the only feasible option due to the inter- and intrapatient physiological fluctuations.

In conclusion, this study did not confirm clearance of CSF tracer to the nasal mucosa in humans, unlike in many previous animal studies, thus questioning the importance of this molecular clearance route in humans. The method applied in this study has limitations, particularly concerning the use of a small molecular weight CSF tracer. Future studies are needed to address to which extent molecular efflux to nasal mucosa differ between animals and humans.

\section{Methods}

Subjects and study design. The study design was prospective and observational. Patients referred to the Department of neurosurgery, Oslo University Hospital-Rikshospitalet were included for consecutive MRI exams after intrathecal administration of the contrast agent gadobutrol. Inclusion criteria were tentative CSF circulation disorders. Exclusion criteria were age $<18$ years or $>80$ years, pregnancy and breastfeeding, known adverse reaction to contrast media, history of severe allergic reaction in general, and renal dysfunction. A total number of 34 patients were included for imaging. After imaging, 10 subjects were excluded due to movement artefacts, 
missing MRI exams, or signs of rhinitis at MRI. Of the remaining 24 patients, 12 were included in a previous study assessing tracer enrichment in the parasagittal dura ${ }^{16}$.

MRI was obtained approximately $8 \mathrm{a} . \mathrm{m}$. followed by an X-ray guided lumbar puncture performed by an experienced interventional neuroradiologist. Backflow from the puncture needle verified a correct position in the SAS. A radiopaque contrast agent, typically $3 \mathrm{ml}$ of $270 \mathrm{mg} \mathrm{I} / \mathrm{ml}$ iodixanol (Visipaque, GE Healthcare), was injected to confirm unrestricted passage in the lumbar SAS. Thereafter, $0.5 \mathrm{ml}$ of $1.0 \mathrm{mmol} / \mathrm{ml}$ gadobutrol (Gadovist, Bayer AB, Sweden) was administered. After needle removal, the study subjects were instructed to rotate themselves around the long axis of the body once and remain in a supine position for transportation back to the MRI suite for the first post-contrast image.

MRI protocol. A 3 T Philips Ingenia MRI scanner (Philips Medical Systems) with a 32-channel head coil was used with equal imaging protocol settings at all time points to acquire whole-brain sagittal T1-weighted volume scans with suppression of intraluminal blood signal ("Black Blood") (T1-BB). The parameters were TR/ $\mathrm{TE}=700 / 35 \mathrm{~ms}$, echo train length $=55$, flip angle $=80^{\circ}$, 2 averages, $1 \times 1 \times 1 \mathrm{~mm}$ voxel size (isotropic). Acquisition time was $4 \mathrm{~min}$ and $54 \mathrm{~s}$.

For practical reasons, the consecutive MRI exams could not be obtained at identical time points in every subject. The MRI exams were therefore categorized into the following approximate time points according to an intention-to-scan basis: pre-contrast, $<1 \mathrm{~h}$ (mean $18 \mathrm{~min}, \mathrm{SD} \pm 5 \mathrm{~min}$ ) after contrast, $3 \mathrm{~h}$ (mean $3 \mathrm{~h}$ and $7 \mathrm{~min}$, $\mathrm{SD} \pm 20 \mathrm{~min}$ ) after contrast, $6 \mathrm{~h}$ (mean $6 \mathrm{~h}$ and $2 \mathrm{~min}, \mathrm{SD} \pm 24 \mathrm{~min}$ ) after contrast, $24 \mathrm{~h}$ (mean $25 \mathrm{~h}$ and $2 \mathrm{~min}$, $\mathrm{SD} \pm 1 \mathrm{~h}$ and $13 \mathrm{~min}$ ) after contrast and $48 \mathrm{~h}$ (mean $47 \mathrm{~h}$ and $58 \mathrm{~min}, \mathrm{SD} \pm 1 \mathrm{~h}$ and $25 \mathrm{~min}$ ) after contrast. The patients were instructed to stay in bed until the $6 \mathrm{~h}$ ' time point and were allowed to move freely thereafter.

Whole-brain 3D T1-gradient echo (GRE) images were also obtained during each imaging session. The imaging parameters were as follows: $\mathrm{TR}=$ shortest (typically $5.1 \mathrm{~ms}$ ), echo time= shortest (typically $2.3 \mathrm{~ms}$ ), echo train length $=232$, flip angle $=8^{\circ}, 1$ average, $1 \times 1 \times 1 \mathrm{~mm}$ voxel size and acquisition time $6 \mathrm{~min}$ and $29 \mathrm{~s}$.

T1-BB scans were deemed superior to T1-GRE with regards to having a better signal-to-noise ratio in the region of the nasal mucosa and were chosen for the ROI-analysis in all areas of interest. The T1-GRE images were used for visually detection of tracer inferior to the cribriform plate and in elaboration of the image showing the tracer immediately below the cribriform plate (Fig. 2).

Intrathecal administration of gadolinium. Gadobutrol is approved for intravenous use only by the United States' Food and Drug Administration (FDA). Intrathecal administration of gadobutrol has so far been used off-label in a controlled study setting at our institution in patients with specific clinical indications for work-up of CSF circulation disorders. Gadobutrol is a macrocyclic gadolinium contrast agent. Concerns about gadolinium brain deposition have primarily been associated with linear contrast agents ${ }^{43}$. Recently published data showed the same safety profile for intrathecal administration of gadobutrol as for intrathecal administration of the CT contrast agent iodixanol ${ }^{18}$, and no signs of deposition in brain tissue have been detected after 4 weeks $^{19}$.

Image analysis. All T1-BB volume scans were reconstructed to $1 \mathrm{~mm}$ thick coronal sections perpendicular to a line between the anterior and posterior commissure. A board-certified neuroradiologist (EM) with 7 years' experience in neuroradiology manually placed 24 circular, pre-defined regions of interest (ROIs) at all time points using the hospital's Picture Archiving and Communication System (SECTRA IDS7, Sweden). The placements of the ROIs in the nasal mucosa and the SAS in one patient are illustrated in Fig. 5. The placements of the ROIs in the brain parenchyma can be found in Supplementary Fig. S2.

The ROIs in the nasal turbinates, including the posterior parts, were based on anatomical considerations in rodents ${ }^{2}$, a study of lymphatic vessels in humans ${ }^{44}$, post-mortem studies of humans ${ }^{10}$ and the PET-study by de Leon et al. ${ }^{11}$ to be as anatomically correct as possible for detection of tracer in lymphatic vessels. The lymphatic vessels were expected to be of size far below the image resolution of $1 \mathrm{~mm}$, and therefore not directly detectable.

The exact placement and size of each set of ROIs were chosen with the intention to avoid artefacts and to cover as much relevant tissue as possible and at the same time striving to be anatomically stringent between different time points. 16 of the ROIs were placed in the mucosa/submucosa of the nasal turbinates and nasal septum as follows: 3 ROIs were placed bilaterally on each middle and inferior turbinate including the posterior parts of the turbinates; 1 ROI bilaterally on the superior turbinates; 1 ROI on both sides in the superior part of the nasal septum. 8 ROIs outside of the nasal cavity was measured: bilaterally in the deep white matter of the frontal lobes; bilaterally in straight gyrus of the frontal lobe and the adjacent SAS, respectively.

In addition, ROIs were placed bilaterally in the vitreous body of the ocular bulb for reference (Supplementary Fig. S1). The average pixel intensity for each ROI was measured. Further, we divided the average pixel intensity against the average pixel intensity in the reference ROIs placed within the vitreous body bilaterally in the coronal reconstructed images from the same T1-BB scan. Any significant tracer accumulation in the vitreous body after intrathecal administration was considered highly unlikely. We refer to this method as normalization of pixel intensity; the process corrects for any baseline changes of image greyscale due to image scaling.

Another board-certified neuroradiologist (GR) with 13 years' experience in neuroradiology verified the placement of the ROIs and the overall quality of the images.

The 3D T1-GRE images were visually analysed in reconstructed $1 \mathrm{~mm}$ thick coronal and sagittal images for tracer inferior to the cribriform plate in all 24 patients for all time points. The 3D T1-GRE images were also used as source data for Fig. 2: the pre-contrast images were segmented using SPM12 (The Wellcome Centre for Human Neuroimaging, London, UK) and images from different time points were aligned in 3D slicer (version 4, www.slicer.org $)^{45}$. Figure 2 is composed of a combination of images from one patient: pre-contrast and the 
$48 \mathrm{~h}$ ' time points were subtracted from the 3 and 6 h' timepoints to enhance differences between the images; In this case to visualize the distribution of the tracer 3 and $6 \mathrm{~h}$ after contrast.

Statistics. SPSS software version 24 (IBM Corporation, Armonk, NY, USA) was used for statistical analyses. Linear mixed model analysis was used to assess whether changes in signal unit ratios over time were statistically significant. Pearson Chi-square test was used to determine differences between categorical data. Statistical significance at the 0.05 level (two-tailed) was accepted.

Ethical approvals. The study was approved by the Institutional Board of Oslo University Hospital (2015/1868), the Regional Ethical Committee of South-East Norway (2015/96) and the National Medicines Agency (15/04932-7). The protocol was conducted in accordance with the relevant guidelines and regulations. Oral and written informed consent was obtained from all participants.

Received: 5 March 2020; Accepted: 14 August 2020

Published online: 11 September 2020

\section{References}

1. Kida, S., Pantazis, A. \& Weller, R. O. CSF drains directly from the subarachnoid space into nasal lymphatics in the rat. Anatomy, histology and immunological significance. Neuropathol. Appl. Neurobiol. 19, 480-488. https://doi.org/10.1111/j.1365-2990.1993. tb00476.x (1993).

2. Pizzo, M. E. et al. Intrathecal antibody distribution in the rat brain: Surface diffusion, perivascular transport and osmotic enhancement of delivery. J. Physiol. 596, 445-475. https://doi.org/10.1113/JP275105 (2018).

3. Weller, R. O., Kida, S. \& Zhang, E. T. Pathways of fluid drainage from the brain-morphological aspects and immunological significance in rat and man. Brain Pathol. 2, 277-284. https://doi.org/10.1111/j.1750-3639.1992.tb00704.x (1992).

4. Koh, L., Zakharov, A. \& Johnston, M. Integration of the subarachnoid space and lymphatics: Is it time to embrace a new concept of cerebrospinal fluid absorption?. Cerebrospinal Fluid Res. 2, 6. https://doi.org/10.1186/1743-8454-2-6 (2005).

5. Bradbury, M. W. \& Westrop, R. J. Factors influencing exit of substances from cerebrospinal fluid into deep cervical lymph of the rabbit. J. Physiol. 339, 519-534. https://doi.org/10.1113/jphysiol.1983.sp014731 (1983).

6. Zhang, E. T., Richards, H. K., Kida, S. \& Weller, R. O. Directional and compartmentalised drainage of interstitial fluid and cerebrospinal fluid from the rat brain. Acta Neuropathol. 83, 233-239. https://doi.org/10.1007/bf00296784 (1992).

7. Szentistvanyi, I., Patlak, C. S., Ellis, R. A. \& Cserr, H. F. Drainage of interstitial fluid from different regions of rat brain. Am. J. Physiol. 246, F835-844. https://doi.org/10.1152/ajprenal.1984.246.6.F835 (1984).

8. Nagra, G., Koh, L., Zakharov, A., Armstrong, D. \& Johnston, M. Quantification of cerebrospinal fluid transport across the cribriform plate into lymphatics in rats. Am. J. Physiol. Regul. Integr. Comp. Physiol. 291, R1383-1389. https://doi.org/10.1152/ajpregu.00235 .2006 (2006).

9. McComb, J. G. Recent research into the nature of cerebrospinal fluid formation and absorption. J. Neurosurg. 59, 369-383. https ://doi.org/10.3171/jns.1983.59.3.0369 (1983).

10. Johnston, M., Zakharov, A., Papaiconomou, C., Salmasi, G. \& Armstrong, D. Evidence of connections between cerebrospinal fluid and nasal lymphatic vessels in humans, non-human primates and other mammalian species. Cerebrospinal. Fluid Res. 1, 2. https ://doi.org/10.1186/1743-8454-1-2 (2004).

11. de Leon, M. J. et al. Cerebrospinal fluid clearance in Alzheimer disease measured with dynamic PET. J. Nucl. Med. 58, 1471-1476. https://doi.org/10.2967/jnumed.116.187211 (2017).

12. Louveau, A. et al. Structural and functional features of central nervous system lymphatic vessels. Nature 523, 337-341. https://doi. org/10.1038/nature14432 (2015).

13. Aspelund, A. et al. A dural lymphatic vascular system that drains brain interstitial fluid and macromolecules. J. Exp. Med. 212, 991-999. https://doi.org/10.1084/jem.20142290 (2015).

14. Iliff, J. J. et al. A paravascular pathway facilitates CSF flow through the brain parenchyma and the clearance of interstitial solutes, including amyloid beta. Sci. Transl. Med. 4, 147. https://doi.org/10.1126/scitranslmed.3003748 (2012).

15. Da Mesquita, S. et al. Functional aspects of meningeal lymphatics in ageing and Alzheimer's disease. Nature 560, 185-191. https ://doi.org/10.1038/s41586-018-0368-8 (2018).

16. Ringstad, G. \& Eide, P. K. Cerebrospinal fluid tracer efflux to parasagittal dura in humans. Nat. Commun. 11, 354 . https://doi. org/10.1038/s41467-019-14195-x (2020).

17. Ringstad, G., Vatnehol, S. A. S. \& Eide, P. K. Glymphatic MRI in idiopathic normal pressure hydrocephalus. Brain 140, $2691-2705$. https://doi.org/10.1093/brain/awx191 (2017).

18. Edeklev, C. S. et al. Intrathecal use of gadobutrol for glymphatic MR imaging: Prospective safety study of 100 patients. Am. J. Neuroradiol. 40, 1257-1264. https://doi.org/10.3174/ajnr.A6136 (2019).

19. Ringstad, G. et al. Brain-wide glymphatic enhancement and clearance in humans assessed with MRI. JCI Insight 3, 30. https://doi. org/10.1172/jci.insight.121537 (2018).

20. Eide, P. K. \& Ringstad, G. Delayed clearance of cerebrospinal fluid tracer from entorhinal cortex in idiopathic normal pressure hydrocephalus: A glymphatic magnetic resonance imaging study. J. Cereb. Blood Flow Metab. 39, 1355-1368. https://doi. org/10.1177/0271678X18760974 (2019).

21. Tarasoff-Conway, J. M. et al. Clearance systems in the brain-implications for Alzheimer disease. Nat. Rev. Neurol. 11, 457-470. https://doi.org/10.1038/nrneurol.2015.119 (2015).

22. Ethell, D. W. Disruption of cerebrospinal fluid flow through the olfactory system may contribute to Alzheimer's disease pathogenesis. J. Alzheimers Dis. 41, 1021-1030. https://doi.org/10.3233/JAD-130659 (2014).

23. Hardy, J. \& Selkoe, D. J. The amyloid hypothesis of Alzheimer's disease: Progress and problems on the road to therapeutics. Science 297, 353-356. https://doi.org/10.1126/science.1072994 (2002).

24. Peng, W. et al. Suppression of glymphatic fluid transport in a mouse model of Alzheimer's disease. Neurobiol. Dis. 93, $215-225$. https://doi.org/10.1016/j.nbd.2016.05.015 (2016).

25. Mawuenyega, K. G. et al. Decreased clearance of CNS beta-amyloid in Alzheimer's disease. Science 330, 1774. https://doi. org/10.1126/science.1197623 (2010).

26. Iliff, J. J. et al. Impairment of glymphatic pathway function promotes tau pathology after traumatic brain injury. J. Neurosci. 34, 16180-16193. https://doi.org/10.1523/jneurosci.3020-14.2014 (2014). 
27. Ahn, J. H. et al. Meningeal lymphatic vessels at the skull base drain cerebrospinal fluid. Nature 572, 62-66. https://doi.org/10.1038/ s41586-019-1419-5 (2019).

28. Patel, T. K. et al. Dural lymphatics regulate clearance of extracellular tau from the CNS. Mol. Neurodegener. 14, 11. https://doi. org/10.1186/s13024-019-0312-x (2019).

29. Romanova, L. et al. Energy-dependent transport at dural lymphatic vessels is necessary for A $\beta$ brain clearance in Alzheimer's disease. bioRxiv 20, 427617. https://doi.org/10.1101/427617 (2019).

30. Ma, Q., Ineichen, B. V., Detmar, M. \& Proulx, S. T. Outflow of cerebrospinal fluid is predominantly through lymphatic vessels and is reduced in aged mice. Nat. Commun. 8, 1434. https://doi.org/10.1038/s41467-017-01484-6 (2017).

31. Iliff, J. J. et al. Brain-wide pathway for waste clearance captured by contrast-enhanced MRI. J. Clin. Invest. 123, 1299-1309. https ://doi.org/10.1172/jci67677 (2013).

32. Eide, P. K. \& Ringstad, G. MRI with intrathecal MRI gadolinium contrast medium administration: A possible method to assess glymphatic function in human brain. Acta Radiol. Open 4, 2058460115609635. https://doi.org/10.1177/2058460115609635 (2015).

33. Eide, P. K., Vatnehol, S. A. S., Emblem, K. E. \& Ringstad, G. Magnetic resonance imaging provides evidence of glymphatic drainage from human brain to cervical lymph nodes. Sci. Rep. 8, 7194. https://doi.org/10.1038/s41598-018-25666-4 (2018).

34. Lowhagen, P., Johansson, B. B. \& Nordborg, C. The nasal route of cerebrospinal fluid drainage in man. A light-microscope study. Neuropathol. Appl. Neurobiol. 20, 543-550. https://doi.org/10.1111/j.1365-2990.1994.tb01008.x (1994).

35. Lochhead, J. J. \& Davis, T. P. Perivascular and perineural pathways involved in brain delivery and distribution of drugs after intranasal administration. Pharmaceutics 11, 20. https://doi.org/10.3390/pharmaceutics11110598 (2019).

36. Chapman, C. D. et al. Intranasal treatment of central nervous system dysfunction in humans. Pharm. Res. 30, 2475-2484. https:// doi.org/10.1007/s11095-012-0915-1 (2013).

37. Lochhead, J. J. \& Thorne, R. G. Intranasal delivery of biologics to the central nervous system. Adv. Drug. Deliv. Rev. 64, 614-628. https://doi.org/10.1016/j.addr.2011.11.002 (2012).

38. Sarafoleanu, C., Mella, C., Georgescu, M. \& Perederco, C. The importance of the olfactory sense in the human behavior and evolution. J. Med. Life 2, 196-198 (2009).

39. Harkema, J. R., Carey, S. A. \& Wagner, J. G. The nose revisited: A brief review of the comparative structure, function, and toxicologic pathology of the nasal epithelium. Toxicol. Pathol. 34, 252-269. https://doi.org/10.1080/01926230600713475 (2006).

40. Weed, L. H. Studies on cerebro-spinal fluid. No. III: The pathways of escape from the Subarachnoid Spaces with particular reference to the Arachnoid Villi. J. Med. Res. 31, 51-91 (1914).

41. Greitz, D. \& Hannerz, J. A proposed model of cerebrospinal fluid circulation: Observations with radionuclide cisternography. Am. J. Neuroradiol. 17, 431-438 (1996).

42. Pendolino, A., Lund, V., Nardello, E. \& Ottaviano, G. The nasal cycle: A comprehensive review*. Rhinology 1, 20. https://doi. org/10.4193/RHINOL/18.021 (2018).

43. Taoka, T. \& Naganawa, S. Gadolinium-based contrast media, cerebrospinal fluid and the glymphatic system: Possible mechanisms for the deposition of gadolinium in the brain. Magn. Reson. Med. Sci. 17, 111-119. https://doi.org/10.2463/mrms.rev.2017-0116 (2018).

44. Pan, W. R., Suami, H., Corlett, R. J. \& Ashton, M. W. Lymphatic drainage of the nasal fossae and nasopharynx: Preliminary anatomical and radiological study with clinical implications. Head Neck 31, 52-57. https://doi.org/10.1002/hed.20926 (2009).

45. Fedorov, A. et al. 3D Slicer as an image computing platform for the quantitative imaging network. Magn. Reson. Imaging 30, 1323-1341. https://doi.org/10.1016/j.mri.2012.05.001 (2012).

\section{Acknowledgements}

We would like to thank Øivind Gjertsen, MD, Bård Nedregaard, MD, and Ruth Sletteberg, MD, all interventional neuroradiologists from the Department of Radiology, Oslo University Hospital-Rikshospitalet, who performed the intrathecal injections. We thank the Intervention Centre and Department of Neurosurgery at the Oslo University Hospital-Rikshospitalet for providing valuable support with MR exams and care-taking of the study subjects. Furthermore, we sincerely thank the Nurse Staff and staff at The Hydrocephalus Outward Clinic, Department of Neurosurgery, Oslo University Hospital-Rikshospitalet for their care-taking of all study subjects throughout the examinations. We also thank Tomas Sakinis, MD, radiologist from the Department of Radiology, Oslo University Hospital, for providing Fig. 2. Finally, we thank Are Hugo Pripp, PhD, Department of Biostatistics, Epidemiology and Health Economics, Oslo University Hospital, Oslo, for help with statistical calculations.

\section{Author contributions}

E.M., P.K.E. and G.R. designed and planned this study. E.M. and G.R. analysed the images. E.M., P.K.E., and G.R. interpreted the data. E.M. drafted the first manuscript. E.M., P.K.E. and G.R. reviewed and revised the manuscript. All authors approved the final version of the manuscript.

\section{Competing interests}

Geir Ringstad has received a speaker fee from Bayer AG (Berlin, Germany). The authors declare no other competing interests concerning this research and publication.

\section{Additional information}

Supplementary information is available for this paper at https://doi.org/10.1038/s41598-020-72031-5.

Correspondence and requests for materials should be addressed to P.K.E.

Reprints and permissions information is available at www.nature.com/reprints.

Publisher's note Springer Nature remains neutral with regard to jurisdictional claims in published maps and institutional affiliations. 
(c) (i) Open Access This article is licensed under a Creative Commons Attribution 4.0 International cc) License, which permits use, sharing, adaptation, distribution and reproduction in any medium or format, as long as you give appropriate credit to the original author(s) and the source, provide a link to the Creative Commons licence, and indicate if changes were made. The images or other third party material in this article are included in the article's Creative Commons licence, unless indicated otherwise in a credit line to the material. If material is not included in the article's Creative Commons licence and your intended use is not permitted by statutory regulation or exceeds the permitted use, you will need to obtain permission directly from the copyright holder. To view a copy of this licence, visit http://creativecommons.org/licenses/by/4.0/.

(C) The Author(s) 2020 